\title{
TABLEAUX COMBINATORICS FOR THE ASYMMETRIC EXCLUSION PROCESS AND ASKEY-WILSON POLYNOMIALS
}

\author{
SYLVIE CORTEEL AND LAUREN K. WILLIAMS
}

\begin{abstract}
Introduced in the late 1960's [33, 43, the asymmetric exclusion process (ASEP) is an important model from statistical mechanics which describes a system of interacting particles hopping left and right on a one-dimensional lattice of $n$ sites. It has been cited as a model for traffic flow and protein synthesis. In the most general form of the ASEP with open boundaries, particles may enter and exit at the left with probabilities $\alpha$ and $\gamma$, and they may exit and enter at the right with probabilities $\beta$ and $\delta$. In the bulk, the probability of hopping left is $q$ times the probability of hopping right. The first main result of this paper is a combinatorial formula for the stationary distribution of the ASEP with all parameters general, in terms of a new class of tableaux which we call staircase tableaux. This generalizes our previous work [14, 15] for the ASEP with parameters $\gamma=\delta=0$. Using our first result and also results of Uchiyama-Sasamoto-Wadati [48, we derive our second main result: a combinatorial formula for the moments of Askey-Wilson polynomials. Since the early 1980's there has been a great deal of work giving combinatorial formulas for moments of various other classical orthogonal polynomials (e.g. Hermite, Charlier, Laguerre, Meixner). However, this is the first such formula for the Askey-Wilson polynomials, which are at the top of the hierarchy of classical orthogonal polynomials.
\end{abstract}

\section{CONTEnTs}

1. Introduction

2. The asymmetric exclusion process (ASEP)

3. Staircase tableaux and the stationary distribution of the ASEP 6

4. Askey-Wilson polynomials and a formula for their moments 8

5. A more flexible Matrix Ansatz 10

6. The proof of the stationary distribution 12

7. The proof of our Askey-Wilson moment formula 22

8. Open problems 24

9. Appendix: Staircase, permutation, and alternative tableaux 25

References $\quad 26$

2000 Mathematics Subject Classification. Primary 05E10; Secondary 82B23, $60 \mathrm{C} 05$.

Key words and phrases. permutation tableaux, asymmetric exclusion process, Matrix Ansatz, staircase tableaux, Askey-Wilson polynomial.

Both authors were partially supported by the grants ANR blanc Gamma and ANR-08-JCJC0011, and the second author was partially supported by the NSF grant DMS-0854432 and an Alfred Sloan Fellowship. 


\section{INTRODUCTION}

The asymmetric exclusion process (ASEP) is an important model from statistical mechanics which was introduced independently in the context of biology [33] and in mathematics [43] around 1970. Since then there has been a huge amount of activity on the ASEP and its variants for a number of reasons: although the definition of the model is quite simple, the ASEP is surprisingly rich. For example, it exhibits boundary-induced phase transitions, spontaneous symmetry breaking, and phase separation. Furthermore, the ASEP is regarded as a primitive model for translation in protein synthesis [33], traffic flow [39], and formation of shocks [19]; it also appears in a kind of sequence alignment problem in computational biology [9]. Important mathematical techniques used to understand this model include the Matrix Ansatz of Derrida, Evans, Hakim, and Pasquier [18], and also the Bethe Ansatz [16, 26, 42].

This paper concerns the ASEP on a one-dimensional lattice of $n$ sites with open boundaries. Particles may enter from the left at rate $\alpha d t$ and from the right at rate $\delta d t$; they may exit the system to the right at rate $\beta d t$ and to the left at rate $\gamma d t$. The probability of hopping left and right is $q d t$ and $u d t$, respectively 1

Dating back to at least 1982, it was realized that there were connections between this model and combinatorics - for example, Young diagrams appeared in [40], and Catalan numbers arose in the analyses of the stationary distribution of the ASEP in [17, 18]. More recently, beginning with papers of Brak and Essam [7], and Duchi and Schaeffer [20], and followed by a number of works including [1, 8, 11, 14, 15, 50], there has been a push to understand the roots of the combinatorial features of the ASEP. Ideally, the goal is to find a combinatorial description of the stationary distribution: that is, to express each component of the stationary distribution as a generating function for a set of combinatorial objects. Up to now, the best result in this direction was provided by [14, 15], which addressed this question when $\gamma=\delta=0$ in terms of permutation tableaux, combinatorial objects introduced in the context of total positivity on the Grassmannian [36].

There is a second reason why combinatorialists became intrigued by the ASEP. Papers of Sasamoto [38] and subsequently Uchiyama, Sasamoto, and Wadati [48] linked the ASEP with open boundaries to orthogonal polynomials, in particular, to the Askey-Wilson polynomials. The Askey-Wilson polynomials are orthogonal polynomials that sit at the top of the hierarchy of (basic) hypergeometric orthogonal polynomials [2, 25, 32], in the sense that all other polynomials in this hierarchy are limiting cases or specializations of the Askey-Wilson polynomials. It is known that orthogonal polynomials have many combinatorial features: indeed, starting around the early 1980's, mathematicians including Flajolet [23], Viennot [49], and Foata [24], initiated a combinatorial approach to orthogonal polynomials. Since then, combinatorial formulas have been given for the moments of (the weight functions of)

\footnotetext{
${ }^{1}$ Actually there is no loss of generality in setting $u=1$, so we will often do so.
} 
many of the polynomials in the Askey scheme, including $q$-Hermite, Chebyshev, $q$ Laguerre, Charlier, Meixner, and Al-Salam-Chihara polynomials, see e.g. [27, 28, 30, 34, 41. However, no such formula was known for the moments of the AskeyWilson polynomials. Therefore when the paper [48] provided a close link between the moments of the Askey-Wilson polynomials and the stationary distribution of the ASEP, this gave the idea that a complete combinatorial understanding of the ASEP might also give rise to the combinatorics of the Askey-Wilson moments.

In this paper we give a complete solution to both of the above problems. Namely, we introduce some new combinatorial objects, which we call staircase tableaux, and we prove that generating functions for staircase tableaux describe the stationary distribution of the ASEP, with all parameters general. We then use this result together with [48] to give a combinatorial formula for the moments of the AskeyWilson polynomials.

The method of proof for our stationary distribution result builds upon important work of Derrida, Evans, Hakim, and Pasquier [18], who introduced a Matrix Ansatz as a tool for understanding its stationary distribution. Briefly, the Matrix Ansatz says that if one can find matrices and vectors satisfying certain relations (the DEHP algebra), then each component of the stationary distribution of the ASEP can be expressed in terms of certain products of these matrices and vectors. Knowing this Ansatz, the strategy for proving our stationary distribution result which one would like to employ is the following: find matrices and vectors satisfying the DEHP algebra, and show that appropriate products enumerate staircase tableaux.

However, it has been known since 1996 [21, Section IV] that when the parameters of the ASEP satisfy certain relations (for example $\alpha \beta=q^{i} \gamma \delta$ ), there is no representation of the DEHP algebra. Therefore the above strategy cannot succeed when all parameters of the ASEP are general. What we do instead is to introduce a slight generalization of the Matrix Ansatz (see Theorem 5.2), which is more flexible, albeit harder to use: instead of checking three identities, one must check three infinite families of identites. So our strategy is to find matrices and vectors such that appropriate products enumerate staircase tableaux, and prove that they satisfy the relations of the Generalized Matrix Ansatz. This second step is quite involved, as our "matrices" and "vectors" are somewhat complicated (they have four and two indices each, see Section 6.1), and there is no easy way to use induction to prove the three infinite families of identities.

We believe that our new staircase tableaux deserve further study, because of their combinatorial interest and their potential connection to geometry. For example, staircase tableaux of size $n$ have cardinality $4^{n} n$ !, and hence are in bijection with doubly-signed permutations. In [13], we prove this with an explicit bijection, and also develop connections to other combinatorial objects, including matchings, permutations, and trees. Furthermore, because of the connection to the ASEP, we know that our staircase tableaux have some hidden symmetries which are not at all apparent from their definition. For instance, it is clear from the definition of the ASEP that 
the model remains unchanged if we reflect it over the $y$-axis, and exchange parameters $\alpha$ and $\delta, \beta$ and $\gamma$, and $q$ and $u$. However, the corresponding bijection on the level of tableaux has so far eluded us. Finally, staircase tableaux generalize permutation tableaux, which index certain cells in the non-negative part of the Grassmannian [36]; it would be interesting to better understand the relationship between the tableaux, the ASEP, and the geometry, and potentially generalize it to staircase tableaux.

It is worth mentioning that in recent years there has been an explosion of activity [3, 5, 22, 6, 37, 4, 45, 46, 47] surrounding another version of the ASEP, in which particles hop not on a finite lattice but on $\mathbb{Z}$. Much of this interest was inspired by Johansson's work [29], which reinterprets the TASEP (totally asymmetric exclusion process) as a randomly growing Young diagram. Johansson then used the well-understood combinatorics of Young diagrams and semi-standard tableaux to approach the problem of current fluctuations in the TASEP. In light of this, one may hope that a better understanding of the combinatorics of staircase tableaux could lead to even more results on the ASEP with open boundaries.

The structure of this paper is as follows. In Section 2 we define the ASEP (with open boundaries), and in Sections 3 and 4 we state our main results on the ASEP and on Askey-Wilson polynomials. In Section 5 we prove a generalized Matrix Ansatz, and in Sections 6 and 7 we prove our results on the ASEP and Askey-Wilson polynomials. Section 8 gives open problems, and the Appendix describes how staircase tableaux generalize permutation tableaux and alternative tableaux [50].

Acknowledgments: We would like to thank Ira Gessel, Richard Stanley, and Jan de Gier for interesting remarks. We are also grateful to the referees for their thoughtful comments, which helped us to greatly improve the exposition. Finally, we are grateful to Svante Janson, who found a gap in the proof of Proposition 6.11 in the published version of this article; the problem was that to deduce (17) from (16), the old proof used identity $2 E_{j, m+1}^{n}$ rather than identity $2 E_{j, m}^{n}$, as originally stated. This version of the paper corrects the error, by giving a direct proof of Theorem 6.9 (2) (using the number of the published version). We are also grateful to Pawel Hitczenko for useful comments.

\section{The ASYMmetric EXCLUSION PROCESS (ASEP)}

The ASEP is often defined using a continuous time parameter [18]. However, one can also define it as a discrete-time Markov chain, as we will do below. The continuous and discrete-time definitions are equivalent in the sense that their stationary distributions are the same [20].

Definition 2.1. Let $\alpha, \beta, \gamma, \delta, q$, and $u$ be constants such that $0 \leq \alpha \leq 1,0 \leq \beta \leq 1$, $0 \leq \gamma \leq 1,0 \leq \delta \leq 1,0 \leq q \leq 1$, and $0 \leq u \leq 1$. Let $B_{n}$ be the set of all $2^{n}$ words in the language $\{\circ, \bullet\}^{*}$. The ASEP is the Markov chain on $B_{n}$ with transition probabilities: 
- If $X=A \bullet \circ B$ and $Y=A \circ \bullet B$ then $P_{X, Y}=\frac{u}{n+1}$ (particle hops right) and $P_{Y, X}=\frac{q}{n+1}$ (particle hops left).

- If $X=\circ B$ and $Y=\bullet B$ then $P_{X, Y}=\frac{\alpha}{n+1}$ (particle enters from the left).

- If $X=B \bullet$ and $Y=B \circ$ then $P_{X, Y}=\frac{\beta}{n+1}$ (particle exits to the right).

- If $X=\bullet B$ and $Y=\circ B$ then $P_{X, Y}=\frac{\gamma}{n+1}$ (particle exits to the left).

- If $X=B \circ$ and $Y=B \bullet$ then $P_{X, Y}=\frac{\delta}{n+1}$ (particle enters from the right).

- Otherwise $P_{X, Y}=0$ for $Y \neq X$ and $P_{X, X}=1-\sum_{X \neq Y} P_{X, Y}$.

Note that we will sometimes denote a state of the ASEP as a word in $\{0,1\}^{n}$ and sometimes as a word in $\{0, \bullet\}^{n}$. In these notations, the symbols 1 and $\bullet$ denote a particle, while 0 and $\circ$ denote the absence of a particle, which one can also think of as a white particle.

See Figure 1 for an illustration of the four states, with transition probabilities, for the case $n=2$. The probabilities on the loops are determined by the fact that the sum of the probabilities on all outgoing arrows from a given state must be 1 .

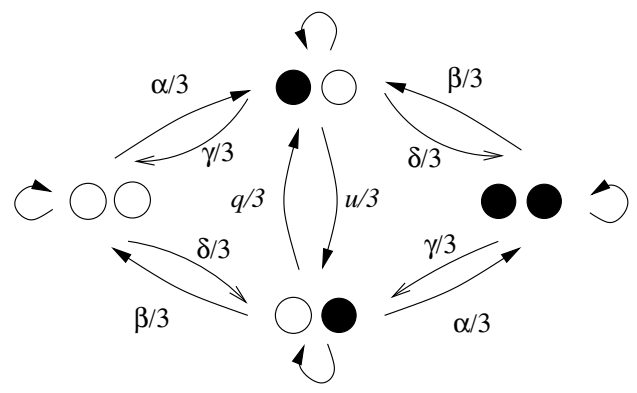

Figure 1. The state diagram of the ASEP for $n=2$

In the long time limit, the system reaches a steady state where all the probabilities $P_{n}\left(\tau_{1}, \tau_{2}, \ldots, \tau_{n}\right)$ of finding the system in configurations $\left(\tau_{1}, \tau_{2}, \ldots, \tau_{n}\right)$ are stationary. More specifically, the stationary distribution is the unique (up to scaling) eigenvector of the transition matrix of the Markov chain with eigenvalue 1.

The ASEP clearly has multiple symmetries, including the following.

- The "left-right" symmetry: if we reflect the ASEP over the $y$-axis, we get back the same model, except that the parameters $\alpha$ and $\delta, \gamma$ and $\beta$, and $u$ and $q$ are switched.

- The "arrow-reversal" symmetry: if we exchange black and white particles, we get back the same model, except that the parameters $\alpha$ and $\gamma, \beta$ and $\delta$, and $u$ and $q$ are switched.

- The "particle-hole" symmetry: if we compose the above two symmetries, i.e. reflect the ASEP over the $y$-axis and exchange black and white particles, we get back the same model, except that $\alpha$ and $\beta$, and $\gamma$ and $\delta$ are switched.

These symmetries imply results about the stationary distribution.

Observation 2.2. The steady state probabilities satisfy the following identities: 
- $P_{n}\left(\tau_{1}, \ldots, \tau_{n}\right)=\left.P_{n}\left(\tau_{n}, \ldots, \tau_{1}\right)\right|_{\alpha \leftrightarrow \delta, \beta \leftrightarrow \gamma, u \leftrightarrow q}$,

- $P_{n}\left(\tau_{1}, \ldots, \tau_{n}\right)=\left.P_{n}\left(1-\tau_{1}, \ldots, 1-\tau_{n}\right)\right|_{\alpha \leftrightarrow \gamma, \beta \leftrightarrow \delta, u \leftrightarrow q}$,

- $P_{n}\left(\tau_{1}, \ldots, \tau_{n}\right)=\left.P_{n}\left(1-\tau_{n}, \ldots, 1-\tau_{1}\right)\right|_{\alpha \leftrightarrow \beta, \gamma \leftrightarrow \delta}$.

Above, the notation $\left.\right|_{\alpha \leftrightarrow \delta}$ indicates that the parameters $\alpha$ and $\delta$ are exchanged. These symmetries are related to the symmetries of the Askey-Wilson polynomials (see for example Remark 4.1), though neither is a direct consequence of the other.

\section{Staircase tableaux And the stationary Distribution of the ASEP}

The main combinatorial objects of this paper are some new tableaux which we call staircase tableaux. These tableaux generalize permutation tableaux (equivalently, alternative tableaux).

Definition 3.1. A staircase tableau of size $n$ is a Young diagram of "staircase" shape $(n, n-1, \ldots, 2,1)$ such that boxes are either empty or labeled with $\alpha, \beta, \gamma$, or $\delta$, subject to the following conditions:

- no box along the diagonal is empty;

- all boxes in the same row and to the left of a $\beta$ or a $\delta$ are empty;

- all boxes in the same column and above an $\alpha$ or a $\gamma$ are empty.

The type of a staircase tableau is a word in $\{\bullet, \circ\}^{n}$ obtained by reading the diagonal boxes from northeast to southwest and writing $a \bullet$ for each $\alpha$ or $\delta$, and $a \circ$ for each $\beta$ or $\gamma$.

Remark 3.2. For convenience, we sometimes refer to the type of a staircase tableau as a word in $\{D, E\}^{n}$ rather than $\{\bullet, \circ\}^{n}$, by identifying $\circ$ by $E$ and $\bullet$ by $D$.

See the left of Figure 2 for an example of a staircase tableau.
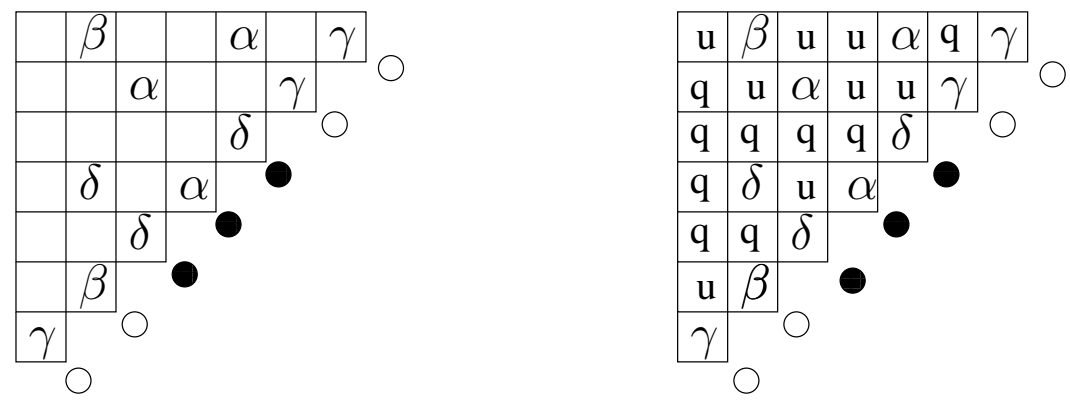

Figure 2. A staircase tableau of size 7 and type $\circ \circ \bullet \bullet \bullet \circ \circ$

Definition 3.3. The weight $\operatorname{wt}(\mathcal{T})$ of a staircase tableau $\mathcal{T}$ is a monomial in $\alpha, \beta, \gamma, \delta, q$, and $u$, which we obtain as follows. Every blank box of $\mathcal{T}$ is assigned a $q$ or $u$, based on the label of the closest labeled box to its right in the same row and the label of the closest labeled box below it in the same column, such that:

- every blank box which sees a $\beta$ to its right gets assigned a u; 
- every blank box which sees a $\delta$ to its right gets assigned a $q$;

- every blank box which sees an $\alpha$ or $\gamma$ to its right, and an $\alpha$ or $\delta$ below it, gets assigned a $u$;

- every blank box which sees an $\alpha$ or $\gamma$ to its right, and a $\beta$ or $\gamma$ below it, gets assigned a $q$.

After assigning a $q$ or $u$ to each blank box in this way, the weight of $\mathcal{T}$ is then defined as the product of all labels in all boxes.

The right of Figure 2 shows that this staircase tableau has weight $\alpha^{3} \beta^{2} \gamma^{3} \delta^{3} q^{9} u^{8}$.

Remark 3.4. The weight of a staircase tableau always has degree $n(n+1) / 2$. For convenience, we will sometimes set $u=1$, since this results in no loss of information.

Our first main result (to be proved in Section [6) is the following.

Theorem 3.5. Consider any state $\tau$ of the ASEP with $n$ sites, where the parameters $\alpha, \beta, \gamma, \delta, q$ and $u$ are general. Set $Z_{n}=\sum_{\mathcal{T}} \mathrm{wt}(\mathcal{T})$, where the sum is over all staircase tableaux of size $n$. Then the steady state probability that the ASEP is at state $\tau$ is precisely

$$
\frac{\sum_{\mathcal{T}} \mathrm{wt}(\mathcal{T})}{Z_{n}}
$$

where the sum is over all staircase tableaux $\mathcal{T}$ of type $\tau$. In particular, $Z_{n}$ is the partition function for the ASEP.

Figure 3 illustrates Theorem 3.5 for the state $\bullet \bullet$ of the ASEP. All staircase tableaux $\mathcal{T}$ of type $\bullet \bullet$ are shown. It follows that the steady state probability of $\bullet \bullet$ is

$$
\begin{aligned}
& \frac{\alpha^{2} u+\delta^{2} q+\alpha \delta q+\alpha \delta u+\alpha^{2} \delta+\alpha \beta \delta+\alpha \gamma \delta+\alpha \delta^{2}}{Z_{2}} .
\end{aligned}
$$

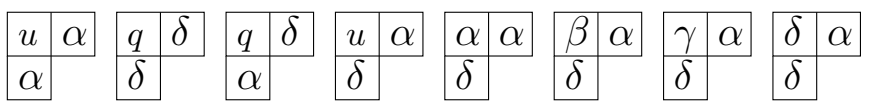

Figure 3. The tableaux of type

We can also obtain combinatorial formulas for various physical quantities. Theorem 3.6 below will be proved in Section 6.4

Theorem 3.6. Consider the ASEP with $n$ sites. Then we have the following:

- The current in the steady state is $\frac{Z_{n-1}\left(\alpha \beta-\gamma \delta q^{n-1}\right)}{Z_{n}}$, where $Z_{n}$ is the generating function for staircase tableaux of size $n$.

- The average particle number $\left\langle\tau_{i}\right\rangle_{n}$ at site $i$ is given by $Z_{n}^{-1}$ times the generating function for all staircase tableaux of size $n$ which have an $\alpha$ or $\delta$ at the ith position along the diagonal.

- Similarly, the m-point function $\left\langle\tau_{i_{1}} \ldots \tau_{i_{m}}\right\rangle_{n}$ is given by $Z_{n}^{-1}$ times the generating function for all staircase tableaux of size $n$ which have an $\alpha$ or $\delta$ in positions $i_{1}, i_{2}, \ldots$, and $i_{m}$ along the diagonal. 
Remark 3.7. In [14] and [15], which concerned the ASEP with parameters $\gamma=$ $\delta=0$, we gave combinatorial expressions for the stationary distribution in terms of permutation tableaux: more specifically, the steady state probability of a state $\tau$ was given by enumerating permutation tableaux lying in a Young diagram of shape $\lambda(\tau)$, according to the number of 1 's in the top row and the number of unrestricted rows.

Subsequently [10] and [12] observed that a permutation tableau is determined by complementary statistics, namely the positions of the topmost 1 's not in the first row, and rightmost restricted zero's. Independently, Viennot [50] defined some new alternative tableaux, in order to give a simpler formula for the stationary distribution of the ASEP when $\gamma=\delta=0$ than the one in [14, 15] ; it turns out that alternative tableaux can be obtained from permutation tableaux, by making boxes red, blue, or empty, based on whether the box of the associated permutation tableau contains a topmost 1, rightmost restricted zero, or neither. Our staircase tableaux generalize both kinds of tableaux, but now have non-empty boxes labeled by $\alpha, \beta, \gamma, \delta$. Additionally, instead of working with Young diagrams of various shapes, we now always work with a staircase shape, whose type encodes the same information (namely, the corresponding state of the ASEP) that the shape used to. Working with this larger shape seems to be the only natural way to assign the appropriate powers of $q$ and $u$ to the tableau.

Remark 3.8. As an alternative to Definition 3.3, suppose we define the dual weight $\mathrm{wt}^{\prime}(\mathcal{T})$ of a staircase tableau by taking the product of all labels in all boxes, after having filled the blank boxes of $\mathcal{T}$ according to the following rule:

- every blank box which sees an $\alpha$ below it gets assigned a u;

- every blank box which sees a $\gamma$ below it gets assigned a q;

- every blank box which sees an $\alpha$ or $\delta$ to its right, and a $\beta$ or $\delta$ below it, gets assigned a q;

- every blank box which sees a $\beta$ or $\gamma$ to its right, and a $\beta$ or $\delta$ below it, gets assigned a $u$.

Then Theorem 3.5 continues to hold, with wt replaced by $\mathrm{wt}^{\prime}$. This follows from the left-right symmetry of the ASEP. More specifically, note that if $\mathcal{T}$ is a staircase tableau, then the tableau $\mathcal{T}^{\prime}$ obtained by transposing $\mathcal{T}$ then switching $\alpha$ and $\delta$, and $\beta$ and $\gamma$ is still a staircase tableau. Our observation now follows from Theorem 3.5, the fact that $\mathrm{wt}^{\prime}\left(\mathcal{T}^{\prime}\right)=\operatorname{wt}(\mathcal{T})$, and Observation 2.2. (Alternatively, we could have proved the observation using a method analogous to the proof of Theorem 3.5.)

\section{Askey-Wilson POLYNOMIALS AND A FORMUla FOR THEIR MOMENTS}

The Askey-Wilson polynomials are orthogonal polynomials with five free parameters $(a, b, c, d, q)$. They reside at the top of the hierarchy of the one-variable orthogonal polynomial family in the Askey scheme [2, 25, 32]. In this section we define the Askey-Wilson polynomials, following the exposition of [2] and [48], then state a combinatorial formula for their moments. 
The $q$-shifted factorial is defined by

$$
\left(a_{1}, a_{2}, \cdots, a_{s} ; q\right)_{n}=\prod_{r=1}^{s} \prod_{k=0}^{n-1}\left(1-a_{r} q^{k}\right)
$$

and the basic hypergeometric function is given by

$$
{ }_{r} \phi_{s}\left[\begin{array}{l}
a_{1}, \cdots, a_{r} \\
b_{1}, \cdots, b_{s}
\end{array} ; q, z\right]=\sum_{k=0}^{\infty} \frac{\left(a_{1}, \cdots, a_{r} ; q\right)_{k}}{\left(b_{1}, \cdots, b_{s}, q ; q\right)_{k}}\left((-1)^{k} q^{k(k-1) / 2}\right)^{1+s-r} z^{k} .
$$

The Askey-Wilson polynomial $P_{n}(x)=P_{n}(x ; a, b, c, d \mid q)$ is explicitly defined by

$$
P_{n}(x)=a^{-n}(a b, a c, a d ; q)_{n{ }_{4}} \phi_{3}\left[\begin{array}{c}
q^{-n}, q^{n-1} a b c d, a e^{i \theta}, a e^{-i \theta} \\
a b, a c, a d
\end{array} ; q, q\right],
$$

with $x=\cos \theta$ for $n \in \mathbb{Z}_{+}:=\{0,1,2, \cdots\}$. It satisfies the three-term recurrence

$$
A_{n} P_{n+1}(x)+B_{n} P_{n}(x)+C_{n} P_{n-1}(x)=2 x P_{n}(x),
$$

with $P_{0}(x)=1$ and $P_{-1}(x)=0$, where

$$
\begin{aligned}
A_{n} & =\frac{1-q^{n-1} a b c d}{\left(1-q^{2 n-1} a b c d\right)\left(1-q^{2 n} a b c d\right)}, \\
B_{n} & =\frac{q^{n-1}}{\left(1-q^{2 n-2} a b c d\right)\left(1-q^{2 n} a b c d\right)}\left[\left(1+q^{2 n-1} a b c d\right)\left(q s+a b c d s^{\prime}\right)-q^{n-1}(1+q) a b c d\left(s+q s^{\prime}\right)\right], \\
C_{n} & =\frac{\left(1-q^{n}\right)\left(1-q^{n-1} a b\right)\left(1-q^{n-1} a c\right)\left(1-q^{n-1} a d\right)\left(1-q^{n-1} b c\right)\left(1-q^{n-1} b d\right)\left(1-q^{n-1} c d\right)}{\left(1-q^{2 n-2} a b c d\right)\left(1-q^{2 n-1} a b c d\right)},
\end{aligned}
$$

$$
\text { and } s=a+b+c+d, \quad s^{\prime}=a^{-1}+b^{-1}+c^{-1}+d^{-1} .
$$

Remark 4.1. It is obvious from the three-term recurrence that the polynomials $P_{n}(x)$ are symmetric in $a, b, c$ and $d$.

For $|a|,|b|,|c|,|d|<1$, using $z=e^{i \theta}$, the orthogonality is expressed by

$$
\oint_{C} \frac{d z}{4 \pi i z} w\left(\frac{z+z^{-1}}{2}\right) P_{m}\left(\frac{z+z^{-1}}{2}\right) P_{n}\left(\frac{z+z^{-1}}{2}\right)=h_{n} \delta_{m n},
$$

where the integral contour $C$ is a closed path which encloses the poles at $z=a q^{k}, b q^{k}$, $c q^{k}, d q^{k}\left(k \in \mathbb{Z}_{+}\right)$and excludes the poles at $z=\left(a q^{k}\right)^{-1},\left(b q^{k}\right)^{-1},\left(c q^{k}\right)^{-1},\left(d q^{k}\right)^{-1}$ $\left(k \in \mathbb{Z}_{+}\right)$, and where

$$
\begin{aligned}
& w(\cos \theta)=\frac{\left(e^{2 i \theta}, e^{-2 i \theta} ; q\right)_{\infty}}{\left(a e^{i \theta}, a e^{-i \theta}, b e^{i \theta}, b e^{-i \theta}, c e^{i \theta}, c e^{-i \theta}, d e^{i \theta}, d e^{-i \theta} ; q\right)_{\infty}}, \\
& \frac{h_{n}}{h_{0}}=\frac{\left(1-q^{n-1} a b c d\right)(q, a b, a c, a d, b c, b d, c d ; q)_{n}}{\left(1-q^{2 n-1} a b c d\right)(a b c d ; q)_{n}}, \\
& h_{0}=\frac{(a b c d ; q)_{\infty}}{(q, a b, a c, a d, b c, b d, c d ; q)_{\infty}} .
\end{aligned}
$$

(In the other parameter region, the orthogonality is continued analytically.) 
The moments are defined by

$$
\mu_{k}=\oint_{C} \frac{d z}{4 \pi i z} w\left(\frac{z+z^{-1}}{2}\right)\left(\frac{z+z^{-1}}{2}\right)^{k} .
$$

The second main result of this paper is a combinatorial formula for the moments of the Askey-Wilson polynomials. In Theorem 4.2 below, we use the substitution

$$
\begin{aligned}
& \alpha=\frac{1-q}{1+a c+a+c}, \quad \beta=\frac{1-q}{1+b d+b+d}, \\
& \gamma=\frac{-(1-q) a c}{1+a c+a+c}, \quad \delta=\frac{-(1-q) b d}{1+b d+b+d},
\end{aligned}
$$

which can be inverted via

$$
\begin{aligned}
& a=\frac{1-q-\alpha+\gamma+\sqrt{(1-q-\alpha+\gamma)^{2}+4 \alpha \gamma}}{2 \alpha} \\
& c=\frac{1-q-\alpha+\gamma-\sqrt{(1-q-\alpha+\gamma)^{2}+4 \alpha \gamma}}{2 \alpha} \\
& b=\frac{1-q-\beta+\delta+\sqrt{(1-q-\beta+\delta)^{2}+4 \beta \delta}}{2 \beta} \\
& d=\frac{1-q-\beta+\delta-\sqrt{(1-q-\beta+\delta)^{2}+4 \beta \delta}}{2 \beta} .
\end{aligned}
$$

Recall that $Z_{\ell}=\sum_{\mathcal{T}} \mathrm{wt}(\mathcal{T})$, where the sum is over all staircase tableaux of size $\ell$.

Theorem 4.2. The $k$ th moment of the Askey-Wilson polynomials is given by

$$
\mu_{k}=h_{0} \sum_{\ell=0}^{k}(-1)^{k-\ell}\left(\begin{array}{l}
k \\
\ell
\end{array}\right)\left(\frac{1-q}{2}\right)^{\ell} \frac{Z_{\ell}}{\prod_{i=0}^{\ell-1}\left(\alpha \beta-\gamma \delta q^{i}\right)} .
$$

\section{A more flexible Matrix Ansatz}

One of the most powerful techniques for studying the ASEP is the so-called Matrix Ansatz, an Ansatz given by Derrida, Evans, Hakim, and Pasquier [18] as a tool for solving for the steady state probabilities $P_{n}\left(\tau_{1}, \ldots, \tau_{n}\right)$ of the ASEP. In this section we will start by recalling their Matrix Ansatz, and then give a new generalization of it which we require for our proof of Theorem 3.5.

For convenience, in this section we set $u=1$. Also, we define unnormalized weights $f_{n}\left(\tau_{1}, \ldots, \tau_{n}\right)$, which are equal to the $P_{n}\left(\tau_{1}, \ldots, \tau_{n}\right)$ up to a constant:

$$
P_{n}\left(\tau_{1}, \ldots, \tau_{n}\right)=f_{n}\left(\tau_{1}, \ldots, \tau_{n}\right) / Z_{n},
$$

where $Z_{n}$ is the partition function $\sum_{\tau} f_{n}\left(\tau_{1}, \ldots, \tau_{n}\right)$. The sum defining $Z_{n}$ is over all possible configurations $\tau \in\{0,1\}^{n}$. Derrida et al showed the following.

Theorem 5.1. [18] Suppose that $D$ and $E$ are matrices, $V$ is a column vector, and $W$ is a row vector, with $W V=1$, such that the following conditions hold:

$$
D E-q E D=D+E, \beta D V-\delta E V=V, \alpha W E-\gamma W D=W .
$$


Then for any state $\tau=\left(\tau_{1}, \ldots, \tau_{n}\right)$ of the ASEP,

$$
f_{n}\left(\tau_{1}, \ldots, \tau_{n}\right)=W\left(\prod_{i=1}^{n}\left(\tau_{i} D+\left(1-\tau_{i}\right) E\right)\right) V .
$$

Note that $\prod_{i=1}^{n}\left(\tau_{i} D+\left(1-\tau_{i}\right) E\right)$ is simply a product of $n$ matrices $D$ or $E$ with matrix $D$ at position $i$ if site $i$ is occupied $\left(\tau_{i}=1\right)$. Also note that Theorem 5.1 implies that $Z_{n}=W(D+E)^{n} V$.

We now state and prove a more flexible version of Theorem 5.1, Our proof generalizes the argument given in [18].

Theorem 5.2. Let $\left\{\lambda_{n}\right\}_{n \geq 0}$ be a family of constants. Let $W$ and $V$ be row and column vectors, with $W V=1$, and let $D$ and $E$ be matrices such that for any words $X$ and $Y$ in $D$ and $E$, we have:

(I) $W X(D E-q E D) Y V=\lambda_{|X|+|Y|+2} W X(D+E) Y V$;

(II) $\beta W X D V-\delta W X E V=\lambda_{|X|+1} W X V$;

(III) $\alpha W E Y V-\gamma W D Y V=\lambda_{|Y|+1} W Y V$.

(Here $|X|$ is the length of $X$.) Then for any state $\tau=\left(\tau_{1}, \ldots, \tau_{n}\right)$ of the ASEP,

$$
f_{n}(\tau)=W\left(\prod_{i=1}^{n}\left(\tau_{i} D+\left(1-\tau_{i}\right) E\right)\right) V .
$$

Proof. We are in the steady state of the ASEP if the net rate of entering each state $\left(\tau_{1}, \ldots, \tau_{n}\right)$ is 0 , or in other words, the following expression equals 0 :

$$
\begin{aligned}
& (-1)^{\tau_{1}}\left(-\alpha f_{n}\left(0, \tau_{2}, \ldots, \tau_{n}\right)+\gamma f_{n}\left(1, \tau_{2}, \ldots, \tau_{n}\right)\right) \\
& +\sum_{i=1}^{n-1}(-1)^{\tau_{i}} \chi\left(\tau_{i} \neq \tau_{i+1}\right)\left(f_{n}\left(\tau_{1}, \ldots, 1,0, \ldots, \tau_{n}\right)-q f_{n}\left(\tau_{1}, \ldots, 0,1, \ldots, \tau_{n}\right)\right) \\
& +(-1)^{\tau_{n}}\left(\beta f_{n}\left(\tau_{1}, \ldots, \tau_{n-1}, 1\right)-\delta f_{n}\left(\tau_{1}, \ldots, \tau_{n-1}, 0\right)\right) .
\end{aligned}
$$

In (3) above, the arguments 1,0 and 0,1 are in positions $i$ and $i+1$, and $\chi$ is the boolean function taking value 1 or 0 based on whether its argument is true or false. So what we have to prove is that the quantities in the right-hand-side of equation (1) satisfy this equation.

By the assumptions (I), (II), and (III) of the theorem, we have the following:

- the expression (2) equals $\pm \lambda_{n} f_{n-1}\left(\tau_{2}, \ldots, \tau_{n}\right)$, based on whether $\tau_{1}$ is 1 or 0 ;

- (3) equals 0 when $\tau_{i}=\tau_{i+1}$; otherwise, based on whether $\tau_{i}$ is 1 or 0 , it equals $\mp \lambda_{n} \sum_{i=1}^{n}\left(f_{n-1}\left(\tau_{1}, \ldots, \hat{\tau}_{i}, \ldots, \tau_{n}\right)+f_{n-1}\left(\tau_{1}, \ldots, \hat{\tau}_{i+1}, \ldots, \tau_{n}\right)\right)$;

- and (4) equals $\mp \lambda_{n} f_{n-1}\left(\tau_{1}, \ldots, \tau_{n-1}\right)$, based on whether $\tau_{n}$ is 1 or 0 .

(Here $\hat{\tau}_{i}$ denotes the omission of the $i$ th component.) Then using these conditions, it is easy to verify that the sum of (2), (3), and (44) is equal to 0 , since all terms involving $f_{n-1}$ cancel out. 


\section{THE PROOF OF THE STATIONARY DISTRIBUTION}

In this section we will prove Theorem 3.5 by: defining vectors $W, V$ and matrices $D, E$; proving that they have the requisite combinatorial interpretation in terms of staircase tableaux; and checking that they satisfy the relations of Theorem 5.2, with $\lambda_{0}=1$ and $\lambda_{n}=\alpha \beta-\gamma \delta q^{n-1}$ for $n \geq 1$.

This is analogous to the proof of [14, Theorem 3.1], albeit much more difficult: in [14], it was obvious that our matrices and vectors satisfied the Matrix Ansatz [14, Lemma 2.5], and easy to show that our combinatorial objects were described by the algebraic relations of the Ansatz, see [14, Figure 6] and the surrounding discussion.

In contrast, in this more general situation, we can give a combinatorial proof of relation (III) of our new Matrix Ansatz, but not for (I) or (II). Instead we give a rather difficult algebraic proof of (I) and (II). First of all, our new "vectors" and "matrices" have two and four indices, respectively, which makes working with them more complicated. Second, to use Theorem 5.2, instead of proving that our vectors and matrices satisfy three identities (as in Theorem 5.1), we must prove that they satisfy three infinite families of identities. Moreover, there is no obvious way to use induction to prove these identities: one cannot take one of the identities and multiply on the left or right to obtain the next identity in the family.

Remark 6.1. In this section we assume $u=1$. Recall that this is no loss of generality, as the weight of a staircase tableau of size $n$ is always a monomial of degree $n(n+1) / 2$.

\subsection{The definition of our matrices.}

Definition 6.2. In what follows, indices range over the non-negative integers. In particular, our matrices and vectors are not finite. We define "row" and "column" vectors $W=\left(W_{i k}\right)_{i, k}$ and $V=\left(V_{j \ell}\right)_{j, \ell}$, and matrices $D=\left(D_{i, j, k, \ell}\right)_{i, j, k, \ell}$ and $E=$ $\left(E_{i, j, k, \ell}\right)_{i, j, k, \ell}$ by the following:

$$
\begin{aligned}
& W_{i k}= \begin{cases}1 & \text { if } i=k=0, \\
0 & \text { otherwise, }\end{cases} \\
& V_{j \ell}=1 \text { always. } \\
& D_{i, j, k, \ell}= \begin{cases}0 & \text { if } j<i \text { or } \ell>k+1, \\
\delta q^{i} & \text { if } i=j-1 \text { and } k=\ell=0, \\
\alpha q^{i} & \text { if } i=j, k=0 \text { and } \ell=1, \\
\delta\left(D_{i, j-1, k-1, \ell}+E_{i, j-1, k-1, \ell}\right)+D_{i, j, k-1, \ell-1} & \text { otherwise. }\end{cases} \\
& E_{i, j, k, \ell}= \begin{cases}0 & \text { if } j<i \text { or } \ell>k+1, \\
\beta q^{i} & \text { if } i=j \text { and } k=\ell=0, \\
\gamma q^{i} & \text { if } i=j, k=0 \text { and } \ell=1, \\
\beta\left(D_{i, j, k-1, \ell}+E_{i, j, k-1, \ell}\right)+q E_{i, j, k-1, \ell-1} & \text { otherwise. }\end{cases}
\end{aligned}
$$

By convention, if any subscript $i, j, k$ or $\ell$ is negative, then $D_{i j k \ell}=E_{i j k \ell}=0$. 


\section{Example 6.3.}

$$
\begin{aligned}
E_{0,2,2,0} & =\beta\left(D_{0,2,1,0}+E_{0,2,1,0}\right)+q E_{0,2,1,-1} \\
& =\beta\left(D_{0,2,1,0}+E_{0,2,1,0}\right) \\
& =\beta\left[\delta\left(D_{0,1,0,0}+E_{0,1,0,0}\right)+D_{0,2,0,-1}+\beta\left(D_{0,2,0,0}+E_{0,2,0,0}\right)+q E_{0,2,0,-1}\right] \\
& =\beta \delta\left(D_{0,1,0,0}+E_{0,1,0,0}\right)+\beta^{2}\left(D_{0,2,0,0}+E_{0,2,0,0}\right) \\
& =\beta \delta(\delta+0)+\beta^{2}(0+0)=\beta \delta^{2}
\end{aligned}
$$

Here, we think of the two coordinates $i$ and $k$ as specifying a "row" of a matrix, and the two coordinates $j$ and $\ell$ as specifying a "column" of a matrix. Therefore matrix multiplication is defined by

$$
(M N)_{i, j, k, \ell}=\sum_{a, b} M_{i, a, k, b} N_{a, j, b, \ell}
$$

Note that when $M$ and $N$ are products of $D$ 's and $E$ 's the sum on the right-hand-side is finite. Specifically, if $M$ is a word of length $r$ in $D$ and $E$, then $M_{i, a, k, b}$ is 0 unless $a+b \leq i+k+r$. This can be shown by induction from the definition of $D$ and $E$, or by using the combinatorial interpretation given in Lemma 6.5.

\subsection{The combinatorial interpretation of our matrices in terms of tableaux.}

We say that a row of a staircase tableau $\mathcal{T}$ is indexed by $\beta$ if the leftmost box in that row which is not occupied by a $q$ or $u$ is a $\beta$. Note that every box to the left of that $\beta$ must be a $u$. Similarly we will talk about rows which are indexed by $\delta$; in this case, every box to the left of that $\delta$ must be a $q$. We will also talk about rows which are indexed by $\alpha / \gamma$, which is shorthand for rows which are indexed by $\alpha$ or $\gamma$.

Theorem 6.4. If $X$ is a word in $D$ 's and $E$ 's, then:

- $X_{i j k \ell}$ is the generating function for all ways of adding $|X|$ new columns of type $X$ to a staircase tableau with $i$ rows indexed by $\delta$ and $k$ rows indexed by $\alpha / \gamma$, so as to obtain a new tableau with $j$ rows indexed by $\delta$ and $\ell$ rows indexed by $\alpha / \gamma$.

- $(W X)_{j \ell}$ is the generating function for staircase tableaux of type $X$ which have $j$ rows indexed by $\delta$ and $\ell$ rows indexed by $\alpha / \gamma$ (and hence $|X|-j-\ell$ rows indexed by $\beta$.)

- $W X V$ is the generating function for all staircase tableaux of type $X$.

The main step in proving Theorem 6.4 is the following lemma, which says that the matrices $D$ and $E$ are "transfer matrices" for building staircase tableaux.

Lemma 6.5. $D_{i, j, k, \ell}$ is the generating function for the weights of all possible new columns with an $\alpha$ or $\delta$ in the bottom box that we could add to the left of a staircase tableau with $i$ rows indexed by $\delta$ and $k$ rows indexed by $\alpha$ or $\gamma$, obtaining a new staircase tableau which has $j$ rows indexed by $\delta$ and $\ell$ rows indexed by $\alpha$ or $\gamma$. Similarly for $E_{i, j, k, \ell}$, where the new column has a $\beta$ or $\gamma$ in the bottom box. 
Proof. Let $D_{i j k \ell}^{\prime}$ denote the generating function for all possible new columns with an $\alpha$ or $\delta$ in the bottom box that we could add to the left of a staircase tableau with $i$ rows indexed by $\delta$ and $k$ rows indexed by $\alpha / \gamma$, obtaining a new staircase tableau which has $j$ rows indexed by $\delta$ and $\ell$ rows indexed by $\alpha$ or $\gamma$. We will show that $D_{i j k \ell}^{\prime}=D_{i j k \ell}$ by showing that $D^{\prime}$ satisfies the same recurrences.

Note that $D_{i j k \ell}^{\prime}=0$ if $j<i$ because adding a new column to a staircase tableau never decreases the number of rows indexed by $\delta$. Also $D_{i j k \ell}^{\prime}=0$ if $\ell>k+1$ because when we add a new column we can never increase the number of rows indexed by $\alpha / \gamma$ by more than 1 .

Now suppose that $k=0$. If we are starting from a tableau with $i$ rows indexed by $\delta$ and 0 rows indexed by $\alpha / \gamma$, then the only way to add a new column is to add a column with an $\alpha$ or $\delta$ at the bottom, with all boxes above empty. If we add a $\delta$, then the resulting tableau has $\ell=0$ rows indexed by $\alpha / \gamma$ and $j=i+1$ rows indexed by $\delta$. The weight of the new column will be $\delta q^{i}$. On the other hand, if we add an $\alpha$ at the bottom, then the resulting tableau has $\ell=1$ rows indexed by $\alpha / \gamma$ and $j=i$ rows indexed by $\delta$. The weight of the new column will be $\alpha q^{i}$. From this discussion it follows that $D_{i j k \ell}^{\prime}=\delta q^{i}$ when $j=i+1$ and $k=\ell=0$, and $D_{i j k \ell}^{\prime}=\alpha q^{i}$ when $j=i, k=0$, and $\ell=1$.

In all other situations, we can assume that $k \geq 1$. Suppose that we are adding a new column $C$ with an $\alpha$ or $\delta$ at the bottom to the left of a staircase tableau with $i$ rows indexed by $\delta$ and $k$ rows indexed by $\alpha / \gamma$, so as to create a new tableau $\mathcal{T}$. Consider the lowest box $B$ of $C$ whose row in $\mathcal{T}$ is indexed by an $\alpha$ or $\gamma$ (such a box exists since $k \geq 1$ ). If we fill $B$ with an $\alpha, \beta, \gamma$ or $\delta$, then the bottom box of $C$ must contain a $\delta$. In this case, if we ignore that bottom $\delta$, then our choices for $C$ are exactly the same as our choices would be for adding a new column to the left of a staircase tableau with $i$ rows indexed by $\delta$ and $k-1$ rows indexed by $\alpha / \gamma$. Therefore, filling $B$ with an $\alpha, \beta, \gamma$ or $\delta$ gives us a contribution of $d\left(D^{\prime}+E^{\prime}\right)_{i, j-1, k-1, \ell}$ to our generating function.

On the other hand, if we leave $B$ empty, then this box will get a weight $u=1$ (recall Remark 6.1). Filling the rest of the column $C$ is like adding a new column to a staircase tableau with $i$ rows indexed by $\delta$ and $k-1$ rows indexed by $\alpha / \gamma$. Therefore leaving $B$ empty gives us a contribution of $D_{i, j, k-1, \ell-1}^{\prime}$ to our generating function.

It follows that when $k \geq 1, D_{i j k \ell}^{\prime}=\delta\left(D^{\prime}+E^{\prime}\right)_{i, j-1, k-1, \ell}+D_{i, j, k-1, \ell-1}^{\prime}$.

Similarly, we define $E_{i j k \ell}^{\prime}$ to be the generating function for all possible new columns with a $\beta$ or $\gamma$ in the bottom box that we could add to the left of a staircase tableau with $i$ rows indexed by $\delta$ and $k$ rows indexed by $\alpha / \gamma$, obtaining a new staircase tableau which has $j$ rows indexed by $\delta$ and $\ell$ rows indexed by $\alpha / \gamma$. The proof that $E_{i j k \ell}^{\prime}=E_{i j k \ell}$ is analogous to the proof we gave for $D^{\prime}$.

Proof of Theorem 6.4. The first item follows from Lemma 6.5 and the definition of matrix multiplication. Multiplying at the left by a $W$ has the effect that we start with the empty tableau and then add columns according to $X$ : so $W X_{j \ell}$ is the 
generating function for staircase tableaux of type $X$ which have $j$ rows indexed by $\delta$ and $\ell$ rows indexed by $\alpha / \gamma$. Finally, multiplying $W X_{j \ell}$ on the right by $V$ has the effect of summing over all $\delta$ and $\ell$, so $W X V$ is the generating function for all staircase tableaux of type $X$.

6.3. The proof that our matrices satisfy the Matrix Ansatz. We now prove that our matrices satisfy Theorem [5.2, with $\lambda_{n}=\alpha \beta-\gamma \delta q^{n-1}$ for $n \geq 1$. Relation (III) has a simple combinatorial proof. However, this proof does not work for relation (II), and indeed it will require a lot more work to prove (I) and (II).

Lemma 6.6. Relation (III) of Theorem 5.2 holds.

Proof. Using Theorem 6.4, relation (III) can be reformulated in terms of staircase tableaux. First we rewrite (III) as

$$
\alpha W E Y V+\gamma \delta q^{n-1} W Y V=\gamma W D Y V+\alpha \beta W Y V,
$$

where $n-1=|Y|$. Since a "type E" corner box of a staircase tableau must be either a $\beta$ or $\gamma$, we can rewrite this again as

$$
\alpha W E^{\beta} Y V+\alpha W E^{\gamma} Y V+\gamma \delta q^{n-1} W Y V=\gamma W D^{\alpha} Y V+\gamma W D^{\delta} Y V+\alpha \beta W Y V .
$$

Here $W E^{\beta} Y V$ denotes the generating function for staircase tableaux of type $E Y$, whose northeast corner box is a $\beta$; the terms $W E^{\gamma} Y V, W D^{\alpha} Y V$, and $W D^{\delta} Y V$ are defined analogously. 2

It is now easy to see that $\alpha W E^{\beta} Y V=\alpha \beta W Y V$ and $\gamma W D^{\alpha} Y V=\gamma \delta q^{n-1} W Y V$, since a box labeled $\beta$ must have only empty boxes (weighted $u=1$ ) to its left, and a box labeled $\delta$ must have only empty boxes (weighted $q$ ) to its left. Also, since the rules for the weight of an empty box which sees a $\gamma$ to its right are the same as the rules for the weight of an empty box which sees an $\alpha$ to its right, we have that $\alpha W E^{\gamma} Y V=\gamma W D^{\alpha} Y V$. This proves relation (III).

Lemma 6.7. For any word $Y$ in $D$ and $E$, we have $Y_{i j k \ell}=q^{|Y|} Y_{i-1, j-1, k, \ell}$.

Proof. We use Theorem 6.4. Note that both $Y_{i j k \ell}$ and $Y_{i-1, j-1, k, \ell}$ enumerate the ways of adding $|Y|$ new columns to a staircase tableau $\mathcal{T}$ so as to increase by $j-i$ the number of rows indexed by $\delta$, and to increase by $\ell-k$ the number of rows indexed by $\alpha / \gamma$. The only difference is the initial number of rows indexed by $\delta$. Since $Y_{i j k \ell}$ has one extra initial row indexed by $\delta$, this will contribute $|Y|$ extra empty boxes which all get the weight $q$. Therefore $Y_{i j k \ell}=q^{|Y|} Y_{i-1, j-1, k, \ell}$.

Proposition 6.8. To prove (I) and (II), it suffices to prove the following identities for all non-negative integers $j$ and $\ell$ :

(1) $(W X D E)_{j \ell}=q(W X E D)_{j \ell}+\alpha \beta(W X(D+E))_{j \ell}-\gamma \delta q^{|X|+1}(W X(D+E))_{j-1, \ell}$.

(2) $\beta(W X D)_{j \ell}=\delta(W X E)_{j-1, \ell}+\alpha \beta(W X)_{j, \ell-1}-\gamma \delta q^{|X|}(W X)_{j-1, \ell-1}$.

\footnotetext{
${ }^{2}$ We could have defined matrices $D^{\alpha}, D^{\delta}, E^{\beta}, E^{\gamma}$ so that they have this combinatorial interpretation, and then set $D=D^{\alpha}+D^{\delta}$ and $E=E^{\beta}+E^{\gamma}$.
} 
Proof. We claim the following: if (1) is true, then for any word $Y$ in $D$ 's and $E$ 's, $(W X D E Y)_{j \ell}$ is equal to

$$
q(W X E D Y)_{j \ell}+\alpha \beta(W X(D+E) Y)_{j \ell}-\gamma \delta q^{|X|+|Y|+1}(W X(D+E) Y)_{j-1, \ell} .
$$

To prove the claim, let $Y$ be any word in $D$ and $E$. Then $(W X D E Y)_{j \ell}$ is equal to:

$$
\begin{aligned}
& \sum_{i, k}(W X D E)_{i k} Y_{i j k \ell} \\
= & \sum_{i, k} q(W X E D)_{i k} Y_{i j k \ell}+\alpha \beta(W X(D+E))_{i k} Y_{i j k \ell}-\gamma \delta q^{|X|+1} \sum_{i k}(W X(D+E))_{i-1, k} Y_{i j k l} \\
= & q(W X E D Y)_{j \ell}+\alpha \beta(W X(D+E) Y)_{j \ell}-\gamma \delta q^{|X|+|Y|+1}(W X(D+E) Y)_{j-1, \ell} .
\end{aligned}
$$

To deduce the final equality above, we applied Lemma 6.7 to the last term.

Now note that if we take the equation of the claim, and sum over all $j$ and $\ell$, then we get precisely (I) (since multiplication on the right by $V$ has the effect of summing over all indices). And if we take (2) and sum over all $j$ and $\ell$, we get (II) This completes the proof.

Lemma 6.9. If the identity (2) of Proposition 6.8 holds for all $j$ and $\ell$, then the identity (1) of Proposition 6.8 holds for all $j$ and $\ell$.

Proof. To prove the lemma, note that $(W X D E)_{j \ell}=\sum_{i, k}(W X D)_{i k} E_{i j k \ell}$ equals:

$$
\begin{aligned}
& \frac{\delta}{\beta} \sum_{i, k}(W X E)_{i-1, k} E_{i j k \ell}+\alpha \sum_{i, k}(W X)_{i, k-1} E_{i j k \ell}-\frac{\gamma \delta q^{|X|}}{\beta} \sum_{i, k}(W X)_{i-1, k-1} E_{i j k \ell} \\
&=\quad \frac{q \delta}{\beta}(W X E E)_{j-1, \ell}+\alpha \sum_{i, k}(W X)_{i, k-1}\left(\beta(D+E)_{i, j, k-1, \ell}+q E_{i, j, k-1, \ell-1}\right) \\
& \quad-\frac{\gamma \delta q^{|X|}}{\beta} \sum_{i, k}(W X)_{i-1, k-1}\left(q \beta(D+E)_{i-1, j-1, k-1, \ell}+q^{2} E_{i-1, j-1, k-1, \ell-1}\right) \\
&=\quad q \beta^{-1} \delta(W X E E)_{j-1, \ell}+\alpha \beta(W X(D+E))_{j \ell}+\alpha q(W X E)_{j, \ell-1} \\
& \quad-\gamma \delta q^{|X|+1}(W X(D+E))_{j-1, \ell}-\beta^{-1} \gamma \delta q^{|X|+2}(W X E)_{j-1, \ell-1} \\
&=\quad\left(W(W X E D)_{j \ell}-\alpha q(W X E)_{j, \ell-1}+q \beta^{-1} \gamma \delta q^{|X|+1}(W X E)_{j-1, \ell-1}\right) \\
& \quad+\alpha \beta(W X(D+E))_{j \ell}+\alpha q(W X E)_{j, \ell-1}-\gamma \delta q^{|X|+1}(W X(D+E))_{j-1, \ell} \\
& \quad-\beta^{-1} \gamma \delta q^{|X|+2}(W X E)_{j-1, \ell-1} \\
&=\quad q(W X E D)_{j \ell}+\alpha \beta(W X(D+E))_{j \ell}-\gamma \delta q^{|X|+1}(W X(D+E))_{j-1, \ell .}
\end{aligned}
$$

In the arguments above, to go from (5) to (6), we used Lemma 6.7 to replace $E_{i j k \ell}$ in the first term by $q E_{i-1, j-1, k, \ell}$.

By Proposition 6.8 and Lemma 6.9, to prove Theorem 3.5, it is enough to prove the following. 
Theorem 6.10. For every word $X$ in $D$ and $E$, and all $j, \ell \in \mathbb{Z}_{\geq 0}$, the identity (2) of Proposition 6.8 holds. Equivalently,

$$
\beta(X D)_{0, j, 0, \ell}-\delta(X E)_{0, j-1,0, \ell}-\alpha \beta(X)_{0, j, 0, \ell-1}+q^{|X|} \gamma \delta(X)_{0, j-1,0, \ell-1}=0 .
$$

To prove Theorem 6.10, we will actually prove the following generalization, which reduces to Theorem 6.10 when $k=0$.

Theorem 6.11. For every word $X$ in $D$ and $E$, and all $j, k, \ell \in \mathbb{Z}_{\geq 0}$, we have

$$
\begin{aligned}
\beta(X D)_{0, j, k, \ell}-\delta & (X E)_{0, j-1, k, \ell}-\alpha \beta(X)_{0, j, k, \ell-1}+q^{|X|+k} \gamma \delta(X)_{0, j-1, k, \ell-1} \\
& =(1-q) \sum_{a, b \geq 1} q^{a|X|} \cdot \frac{a}{b} \cdot E_{0, a, k, k-b} \cdot(X)_{0, j-a, k-b, \ell-1} \\
& =(1-q) \sum_{a, b \geq 1} q^{a|X|} \cdot \frac{b-a+1}{b} \cdot D_{0, a, k, k-b} \cdot(X)_{0, j-a, k-b, \ell-1} .
\end{aligned}
$$

We will prove Theorem 6.11, by induction on the length $|X|$ of the word $X$. Before we begin, we first define a special column in a staircase tableau.

Definition 6.12. A column in a staircase tableau is special if it has a $\beta$ on the bottom, and the next Greek letter which appears above it is a $\delta$. (In particular, a special column has at least one $\delta$.)

We also define the notation $F_{i, j, k, \ell}= \begin{cases}\frac{j-i}{k-\ell} \cdot E_{i, j, k, \ell} & \text { if } \ell<k \\ 0 & \text { otherwise. }\end{cases}$

It is then easy to prove the following.

Lemma 6.13. $F_{i, j, k, \ell}$ is the generating function for the weights of all possible new special columns that we could add to the left of a staircase tableau with $i$ rows indexed by $\delta$ and $k$ rows indexed by $\alpha$ or $\gamma$, obtaining a new staircase tableau which has $j$ rows indexed by $\delta$ and $\ell$ rows indexed by $\alpha$ or $\gamma$.

We now turn to the base case of the induction, which is Proposition 6.14 below (when $i=0$ ). In the statement of the proposition, the notation $\mathbb{1}$ is the indicator function whose value is 1 if its argument is true, and 0 otherwise.

Proposition 6.14. The following identity holds for all non-negative $i, j, k, \ell$.

$$
\begin{aligned}
& \beta D_{i, j, k, \ell}-\delta E_{i, j-1, k, \ell}-q^{i} \alpha \beta \mathbb{1}_{i=j} \mathbb{1}_{\ell=k+1}+q^{i+k} \gamma \delta \mathbb{1}_{j=i+1} \mathbb{1}_{\ell=k+1} \\
& =(1-q) \mathbb{1}_{j>i} \mathbb{1}_{k-\ell \geq j-i-1} \cdot \frac{j-i}{k-\ell+1} \cdot E_{i, j, k, \ell-1} \\
& =(1-q) \mathbb{1}_{j>i} \mathbb{1}_{k-\ell \geq j-i-1} \cdot \frac{k-\ell-j+i+2}{k-\ell+1} \cdot D_{i, j, k, \ell-1}
\end{aligned}
$$

Proof. One may prove the proposition by computing the generating functions for $D_{i, j, k, \ell}$ and $E_{i, j, k, \ell}$, using e.g. the techniques from the proof of Proposition 6.16. One may also give a combinatorial proof, which we will illustrate here. 
First note that Lemma 6.14 is obvious when $l=k+1$. Otherwise, we may assume that $\ell \leq k$, in which case $D_{i, j, k, \ell}=D_{i, j, k, \ell}^{\delta}$ and $E_{i, j-1, k, \ell}=E_{i, j-1, k, \ell}^{\beta}$. Therefore in order to show that (9) equals (10), we need to show that $\beta D_{i, j, k, \ell}^{\delta}-\delta E_{i, j-1, k, \ell}^{\beta}=(1-$ q) $F_{i, j, k, \ell-1}$. The top and bottom rows of Figure 4 represent the quantities $\beta D_{i, j, k, \ell}^{\delta}-$ $\delta E_{i, j-1, k, \ell}^{\beta}$ and $(1-q) F_{i, j, k, \ell-1}$, respectively.
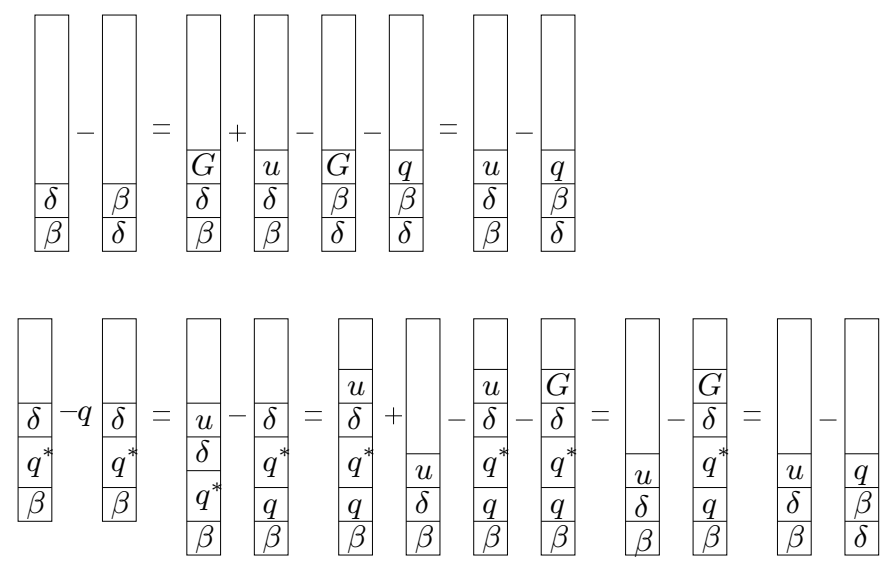

FiguRE 4.

We interpret $\beta D_{i, j, k, \ell}^{\delta}$ as the generating function for columns of type $D_{i, j, k, \ell}^{\delta}$ with an extra $\beta$ added at the bottom. In other words, these are columns of height $k+2$, which contain $j-i \delta$ 's, $(k-\ell)-(j-i)+1 \beta$ 's, which have a $\beta$ at the very bottom (in the first box) and a $\delta$ just above it (in the second box). The third box will contain either a Greek letter (represented by $G$ in the figure) or will be blank, in which case it gets the weight $u$. Similarly, we interpret $\delta E_{i, j-1, k, \ell}^{\beta}$ as the generating function for columns of height $k+2$, which contain $(j-1)-i+1 \delta$ 's, $(k-l)-(j-i-1) \beta$ 's, which have a $\delta$ in the first box, and a $\beta$ in the second box. The third box will contain either a Greek letter, or will be blank, in which case it gets the weight $q$. As illustrated in Figure 4, the generating functions for the two sets of columns which have a Greek letter as their third box are equal, and hence $\beta D_{i, j, k, \ell}^{\delta}-\delta E_{i, j-1, k, \ell}^{\beta}$ represents the signed union of the remaining columns whose third box is blank.

We now consider the quantity $F_{i, j, k, \ell-1}-q F_{i, j, k, \ell-1} \cdot F_{i, j, k, \ell-1}$ is the generating function for columns of height $k+1$, which contain $j-i \delta$ 's, $k-\ell+1-(j-i) \beta$ 's, which have a $\beta$ in the first box, and whose next Greek letter above the $\beta$ is a $\delta$. We insert a new blank box (with weight $u$ ) above the $\delta$, so as to interpret $F_{i, j, k, \ell-1}$ as a generating function for columns of height $k+2$. Inserting a new blank box just above the $\beta$ in the bottom box, we interpret $q F_{i, j, k, \ell-1}$ as the generating function for columns of height $k+2$, which contain $j-i \delta$ 's, $k-\ell+1-(j-i) \beta$ 's, which have a $\beta$ in the first box, then at least one blank box (with weight $q$ ) above that, and whose next Greek letter above the $\beta$ is a $\delta$. We partition the set of columns enumerated by $F_{i, j, k, \ell-1}$ into two parts, based on whether there is at least one blank box above the bottom $\beta$ or not. And we partition the set of columns enumerated by $q F_{i, j, k, \ell-1}$ into 
two parts, based on whether the $\delta$ has a blank box above it or a Greek letter above it. As illustrated in the figure, we get a cancellation among two of the four parts. Finally note that the set of columns with a $\beta$ in the first box, one or more blank boxes above the $\beta$, a $\delta$ above that, and a Greek letter above the $\delta$, have a weight-preserving bijection with the set of columns with a $\delta$ in the first box, a $\beta$ in the second box, and a blank box (with weight $q$ ) in the third box. (Simply move the $\delta$ from the first set of columns to the bottom.) Therefore $\beta D_{i, j, k, \ell}^{\delta}-\delta E_{i, j-1, k, \ell}^{\beta}=(1-q) F_{i, j, k, \ell-1}$.

One may give a similar proof that (9) equals (11), or alternatively check directly that (10) equals (11). This completes the proof of the proposition.

The inductive step of the proof of Theorem 6.11 has two cases: we need to prove that Theorem 6.11 holds for words of the form $E Y$ and also $D Y$, where $Y$ is a word in $D$ and $E$, given that Theorem 6.11 holds for the word $Y$. Our strategy is to explicitly multiply $E$ and $Y$ (respectively $D$ and $Y$ ), expressing $(E Y)_{0, j, k, \ell}$ (respectively $\left.(D Y)_{0, j, k, \ell}\right)$ in terms of quantities such as $(Y)_{0, a, b, c}$. The argument is similar for both cases, so for the sake of brevity, we will include only the proof in the first case. To multiply $E$ and $Y$, we use the following lemma, which follows easily from Lemma 6.7 and the definition of our matrices.

Lemma 6.15. For any word $Z$ in $D$ and $E$ and any non-negative $j, k, \ell$, we have $(E Z)_{0, j, k, \ell}=q^{k} \gamma(Z)_{0, j, k+1, \ell}+\sum_{r, t \geq 0} q^{r|Z|} E_{0, r, k, k-t}(Z)_{0, j-r, k-t, \ell}$

To treat the inductive step, we need to show that the quantity

$$
\begin{gathered}
\beta(E X D)_{0, j, k, \ell}-\delta(E X E)_{0, j-1, k, \ell}-\alpha \beta(E X)_{0, j, k, \ell-1}+q^{|X|+1+k} \gamma \delta(E X)_{0, j-1, k, \ell-1} \\
-(1-q) \sum_{a, b \geq 1} q^{a(|X|+1)} \cdot \frac{a}{b} \cdot E_{0, a, k, k-b} \cdot(E X)_{0, j-a, k-b, \ell-1}
\end{gathered}
$$

equals 0 . We start by applying Lemma 6.15 to the quantities $(E X D)_{0, j, k, \ell},(E X E)_{0, j-1, k, \ell}$, $(E X)_{0, j, k, \ell-1},(E X)_{0, j-1, k, \ell-1}$, and $(E X)_{0, j-a, k-b, \ell-1}$. We obtain

$$
\begin{gathered}
\beta\left(q^{k} \gamma(X D)_{0, j, k+1, \ell}+\sum_{r, t \geq 0} q^{r(|X|+1)} E_{0, r, k, k-t}(X D)_{0, j-r, k-t, \ell}\right) \\
-\delta\left(q^{k} \gamma(X E)_{0, j-1, k+1, \ell}+\sum_{r, t \geq 0} q^{r(|X|+1)} E_{0, r, k, k-t}(X E)_{0, j-r-1, k-t, \ell}\right) \\
-\alpha \beta\left(q^{k} \gamma(X)_{0, j, k+1, \ell-1}+\sum_{r, t \geq 0} q^{r|X|} E_{0, r, k, k-t}(X)_{0, j-r, k-t, \ell-1}\right) \\
+\gamma \delta q^{|X|+1+k}\left(q^{k} \gamma(X)_{0, j-1, k+1, \ell-1}+\sum_{r, t \geq 0} q^{r|X|} E_{0, r, k, k-t}(X)_{0, j-r-1, k-t, \ell-1}\right) \\
-(1-q) \sum_{a, b \geq 1} q^{a(|X|+1)} \frac{a}{b} E_{0, a, k, k-b}\left(q^{k-b} \gamma(X)_{0, j-a, k+1-b, \ell-1}+\right. \\
\left.\sum_{r, t \geq 0} q^{r|X|} E_{0, r, k-b, k-t-b}(X)_{0, j-r-a, k-t-b, \ell-1}\right) .
\end{gathered}
$$


We now apply the inductive hypothesis several times, to rewrite $\beta(X D)_{0, j, k+1, \ell}$, as well as $\beta(X D)_{0, j-r, k-t, \ell}$ for all $r, t$. We obtain the expression

$$
\begin{aligned}
& q^{k} \gamma\left((1-q) \sum_{a, b \geq 1} q^{a|X|}(X)_{0, j-a, k+1-b, \ell-1} \cdot \frac{a}{b} E_{0, a, k+1, k+1-b}\right) \\
& +\sum_{r, t \geq 0} q^{r(|X|+1)} E_{0, r, k, k-t}\left(\alpha \beta(X)_{0, j-r, k-t, \ell-1}-q^{|X|+k-t} \gamma \delta(X)_{0, j-1-r, k-t, \ell-1}+\right. \\
& \left.\quad(1-q) \sum_{a, b \geq 1} q^{a|X|}(X)_{0, j-a-r, k-t-b, \ell-1} \cdot \frac{a}{b} E_{0, a, k-t, k-t-b}\right) \\
& -\alpha \beta \sum_{r, t \geq 0} q^{r|X|} E_{0, r, k, k-t}(X)_{0, j-r, k-t, \ell-1}+q^{|X|+1+k} \gamma \delta \sum_{r, t \geq 0} q^{r|X|} E_{0, r, k, k-t}(X)_{0, j-r-1, k-t, \ell-1} \\
& -(1-q) \sum_{a, b \geq 1} q^{a(|X|+1)} \frac{a}{b} E_{0, a, k, k-b}\left(q^{k-b} \gamma(X)_{0, j-a, k+1-b, \ell-1}+\right. \\
& \left.\sum_{r, t \geq 0} q^{r|X|} E_{0, r, k-b, k-t-b}(X)_{0, j-r-a, k-t-b, \ell-1}\right) .
\end{aligned}
$$

The expression above may be viewed as a linear combination of terms $(X)_{0, v, w, \ell-1}$, where $v \leq j$, and $w \leq k$. To prove that the expression is identically 0 , we will show that the coefficient of each such $(X)_{0, v, w, \ell-1}$ is 0 .

First note that the coefficient of $(X)_{0, j, k-y, \ell-1}$ above (where $y \geq 0$ ) is $E_{0,0, k, k-y} \alpha \beta-$ $\alpha \beta E_{0,0, k, k-y}=0$. Therefore it suffices to analyze the coefficient of each $(X)_{0, j-1-x, k-y, \ell-1}$ for all $x, y \geq 0$. This coefficient is:

$$
\begin{aligned}
& q^{k} \gamma(1-q) q^{(x+1)|X|} \cdot \frac{x+1}{y+1} \cdot E_{0, x+1, k+1, k-y} \\
+ & q^{(x+1)(|X|+1)} \alpha \beta E_{0, x+1, k, k-y} \\
- & q^{(x+1)|X|+x+k-y} \gamma \delta E_{0, x, k, k-y} \\
+ & \sum_{r, t \geq 0} q^{(x+1)|X|+r}(1-q) E_{0, r, k, k-t} \cdot \frac{x+1-r}{y-t} \cdot E_{0, x+1-r, k-t, k-y} \\
- & \alpha \beta q^{(x+1)|X|} E_{0, x+1, k, k-y} \\
& +q^{(x+1)|X|+1+k} \gamma \delta E_{0, x, k, k-y} \\
& -q^{(x+1)|X|+x+k-y}(1-q) \gamma \cdot \frac{x+1}{y+1} \cdot E_{0, x+1, k, k-y-1} \\
- & (1-q) \sum_{r, t \geq 0} q^{(x+1)(|X|+1)-r} E_{0, r, k-y+t, k-y} \cdot \frac{x+1-r}{y-t} \cdot E_{0, x+1-r, k, k-y+t} .
\end{aligned}
$$

Note that every term above contains a factor of $q^{(x+1)|X|}$, which we may delete (since our goal is to show that the sum of the terms is 0 ). If we then combine (13) and (16), (14) and (17), (12) and (15) (using Lemma 6.7), and (마) and (19) (using 
Lemma 6.7), we get

$$
\begin{aligned}
& \left(q^{x+1}-1\right) \alpha \beta E_{0, x+1, k, k-y} \\
+ & \left(q^{k+1}-q^{k+x-y}\right) \gamma \delta E_{0, x, k, k-y} \\
+ & (1-q) \sum_{r \geq 0, t \geq-1} E_{0, r, k, k-t} \cdot \frac{x+1-r}{y-t} \cdot E_{r, x+1, k-t, k-y} \\
- & (1-q) \sum_{r \geq 0, t \geq-1} \frac{x+1-r}{y-t} \cdot E_{0, x+1-r, k, k-y+t} E_{x+1-r, x+1, k-y+t, k-y} .
\end{aligned}
$$

To complete the proof, it suffices to show that the above sum is 0 .

Using the notation $F_{i, j, k, \ell}$ defined earlier, to show that the sum of (20), (21), (22), and (23) vanishes, one may equivalently show the following identity.

Proposition 6.16. For all non-negative $x$ and $y$, we have that

$$
(1-q)(E F-F E)_{0, x+1, k, k-y}=\left(1-q^{x+1}\right) \alpha \beta E_{0, x+1, k, k-y}+\left(q^{k+x-y}-q^{k+1}\right) \gamma \delta E_{0, x, k, k-y} .
$$

Proof. By Lemma 6.13, the equation in Proposition 6.16 has a combinatorial interpretation in terms of columns (and pairs of columns) of staircase tableaux. We prove the equation by computing the generating functions for such columns.

Define the following generating functions:

$$
\begin{aligned}
\mathcal{E}(r, z, s) & =\sum_{j, k, \ell} E_{0, j, k, \ell} r^{j} z^{k} s^{\ell}, \\
\mathcal{E} \mathcal{F}(r, z, s) & =\sum_{j, k, \ell}(E F)_{0, j, k, \ell} r^{j} z^{k} s^{\ell}, \\
\mathcal{F} \mathcal{E}(r, z, s) & =\sum_{j, k, \ell}(F E)_{0, j, k, \ell} r^{j} z^{k} s^{\ell} .
\end{aligned}
$$

where the sum is over all non-negative $j, k, \ell$ such that $\ell \leq k$. Then in order to prove Proposition 6.16, we need to verify that

$(1-q)(\mathcal{E F}(r, z, s)-\mathcal{F} \mathcal{E}(r, z, s))=\alpha \beta \mathcal{E}(r, z, s)-\alpha \beta \mathcal{E}(q r, z, s)+\gamma \delta r \mathcal{E}(q r, z, q s)-\gamma \delta q r \mathcal{E}(r, q z, s)$.

One may compute the three generating functions explicitly by hand. To compute $\mathcal{E}(r, z, s)$, note that since the sum is over $j, k, \ell$ where $\ell \leq k$, we need to enumerate columns with a $\beta$ at the bottom. One may construct such a column from bottom to top: above the $\beta$, there is a non-negative number of blank boxes each with weight $q$. Above these there is an arbitrary sequence of $\beta$ 's, blank boxes, and $\delta$ 's, which we partition into blocks consisting of a $\beta$ at the bottom with some $q$ 's above it, and blocks consisting of a $\delta$ at the bottom with some $u$ 's above it. Above these blocks, there is either an alpha with some $u$ 's above it, or a $\gamma$ with some $q$ 's above it, or nothing. Therefore, since we set $u=1$, we have

$$
\mathcal{E}(r, z, s)=\beta \cdot \frac{1}{1-q z s} \cdot \frac{1}{1-\left(\frac{\beta z}{1-q z s}+\frac{\delta r z}{1-z s}\right)} \cdot\left(\frac{\alpha z s}{1-z s}+\frac{\gamma z s}{1-q z s}+1\right) .
$$


The expressions for the generating functions $\mathcal{E} \mathcal{F}(r, z, s)$ and $\mathcal{F} \mathcal{E}(r, z, s)$ are quite complicated, so we provide a Maple worksheet to compute them and to check the identity relating $\mathcal{E}(r, z, s), \mathcal{E} \mathcal{F}(r, z, s)$ and $\mathcal{F} \mathcal{E}(r, z, s)$. The Maple worksheet may be downloaded at www . math. berkeley. edu/ williams/papers/CW-Identity.zip.

This completes the proof of Theorem 6.11,

Remark 6.17. It would be interesting to find a combinatorial proof of Proposition 6.16 .

6.4. Applications. Once we have a solution to the Matrix Ansatz, it is easy to express physical quantities in terms of matrix products [18]. Set $C=D+E$.

The partition function $Z_{n}$ is written as $W C^{n} V$, and the average particle number at site $i,\left\langle\tau_{i}\right\rangle_{n}$ (where the bracket indicates the average over the stationary probability distribution) is written as

$$
\left\langle\tau_{i}\right\rangle=\frac{W C^{i-1} D C^{n-i} V}{Z_{n}}
$$

Similarly the two-point function $\left\langle\tau_{i} \tau_{j}\right\rangle_{n}$ is given by

$$
\left\langle\tau_{i} \tau_{j}\right\rangle=\frac{W C^{i-1} D C^{j-i-1} D C^{n-j} V}{Z_{n}},
$$

and the $n$-point functions are expressed similarly. The particle current through the bond between the neighboring sites from left to right, which is defined by $J=\left\langle\tau_{i}(1-\right.$ $\left.\left.\tau_{i+1}\right)-q\left(1-\tau_{i}\right) \tau_{i+1}\right\rangle$, is simply given by $J=\frac{Z_{n-1}}{Z_{n}}$. This expression is independent of $i$, as expected in the steady state.

Note that the matrices $D$ and $E$ that we have defined in Section 6.1 actually satisfy the Generalized Matrix Ansatz, not the Matrix Ansatz. However, we can compare quantities computed via the two different Ansatzes using Lemma 17.1, and in particular equation (26). Theorem 3.6 now follows from Theorem 6.4 and the expressions above for the current and $m$-point functions in terms of matrix products.

\section{The PRoOf of OUR ASKey-WiLson MOMENT FORMUlA}

Before proving Theorem 4.2, we need to prove the following result.

Lemma 7.1. Let $D, E, W, V$ be a solution to the Ansatz of Theorem 5.2, and let $\tilde{D}, \tilde{E}, \tilde{W}, \tilde{V}$ be a solution to the Ansatz of Theorem 5.1 . Let h denote the ratio $\frac{\tilde{W} \tilde{V}}{W V}$. Then if $X$ is a word in $D$ and $E$, and $\tilde{X}$ is the corresponding word in $\tilde{D}$ and $\tilde{E}$, then

$$
W X V=h^{-1} \tilde{W} \tilde{X} \tilde{V} \prod_{i=0}^{|X|-1} \lambda_{i} .
$$

In particular, if $\tilde{Z}_{n}=\tilde{W}(\tilde{D}+\tilde{E})^{n} \tilde{V}$, then $Z_{n}=h^{-1} \tilde{Z}_{n} \prod_{i=0}^{n-1} \lambda_{i}$. 
Proof. Let $\tau$ denote the type of $X$, and let $n=|X|$. We use induction on $n$. By Theorem 5.2 and Theorem 5.1 respectively, $W X V$ and $\tilde{W} \tilde{X} \tilde{V}$ compute (unnormalized) steady state probabilities of being in state $\tau$. Therefore $W X V=c_{n} \tilde{W} \tilde{X} \tilde{V}$ for some constant $c_{n}$ that depends on $n$ but not $X$. We want to show that $c_{n}=h^{-1} \prod_{i=0}^{n-1} \lambda_{i}$.

Since we have assumed that $D, E, W, V$ satisfy the relations of Theorem 5.2, $\gamma W D X V-\alpha W E X V=\lambda_{n} W X V$. By induction, we conclude that

$$
\gamma W D X V-\alpha W E X V=\lambda_{n} \tilde{W} \tilde{X} \tilde{V} h^{-1} \prod_{i=0}^{n-1} \lambda_{i} .
$$

But also

$$
\begin{aligned}
\gamma W D X V-\alpha W E X V & =c_{n+1} \gamma \tilde{W} \tilde{D} \tilde{X} \tilde{V}-c_{n+1} \alpha \tilde{W} \tilde{E} \tilde{X} \tilde{V} \\
& =c_{n+1}(\gamma \tilde{W} \tilde{D} \tilde{X} \tilde{V}-\alpha \tilde{W} \tilde{E} \tilde{X} \tilde{V}) \\
& =c_{n+1} \tilde{W} \tilde{X} \tilde{V}
\end{aligned}
$$

by Theorem 5.1. This shows that $c_{n+1}=h^{-1} \prod_{i=0}^{n} \lambda_{i}$, which completes the proof.

We now prove Theorem 4.2, using some results of [48].

Proof of Theorem 4.2. Let $\tilde{Z}_{n}$ denote the partition function from [48, i.e. $\tilde{Z}_{n}=$ $\tilde{W}(\tilde{D}+\tilde{E})^{n} \tilde{V}$, where $\tilde{D}, \tilde{E}, \tilde{W}, \tilde{V}$ are a solution to the Ansatz of Theorem 5.1, and $\tilde{W} \tilde{V}=h_{0}$ (see [48, (4.18)]). Here $h_{0}$ is as in Section 4, Then by [48, Section 6.1],

$$
\tilde{Z}_{n}=\oint_{C} \frac{d z}{4 \pi i z} w\left(\left(z+z^{-1}\right) / 2\right)\left[\frac{z+z^{-1}+2}{1-q}\right]^{n} .
$$

Therefore

$$
\tilde{Z}_{n}=\oint_{C} \frac{d z}{4 \pi i z} w\left(\left(z+z^{-1}\right) / 2\right)\left(\frac{2}{1-q}\right)^{n}\left[\frac{z+z^{-1}}{2}+1\right]^{n},
$$

which implies that

$$
\begin{aligned}
\left(\frac{1-q}{2}\right)^{n} \tilde{Z}_{n} & =\oint_{C} \frac{d z}{4 \pi i z} w\left(\left(z+z^{-1}\right) / 2\right)\left[\frac{z+z^{-1}}{2}+1\right]^{n} \\
& =\sum_{k=0}^{n}\left(\begin{array}{l}
n \\
k
\end{array}\right) \oint_{C} \frac{d z}{4 \pi i z} w\left(\left(z+z^{-1}\right) / 2\right)\left[\frac{z+z^{-1}}{2}\right]^{k} \\
& =\sum_{k=0}^{n}\left(\begin{array}{l}
n \\
k
\end{array}\right) \mu_{k} .
\end{aligned}
$$

Inverting this, we get

$$
\mu_{k}=\sum_{n=0}^{k}(-1)^{k-n}\left(\begin{array}{l}
k \\
n
\end{array}\right)\left(\frac{1-q}{2}\right)^{n} \tilde{Z}_{n} .
$$


By Theorem [3.5, we know that $Z_{n}$ is the generating function for all staircase tableaux of size $n$. By Lemma 7.1,

$$
Z_{n}=h_{0}^{-1} \tilde{Z}_{n} \prod_{i=0}^{n-1}\left(\alpha \beta-\gamma \delta q^{i}\right)
$$

Therefore

$$
\mu_{k}=h_{0} \sum_{n=0}^{k}(-1)^{k-n}\left(\begin{array}{l}
k \\
n
\end{array}\right)\left(\frac{1-q}{2}\right)^{n} \frac{Z_{n}}{\prod_{i=0}^{n-1}\left(\alpha \beta-\gamma \delta q^{i}\right)}
$$

\section{Open PROBLEMS}

8.1. Symmetries in the ASEP. Recall that the ASEP has "left-right," "arrowreversal," and "particle-hole" symmetries, which imply Observation 2.2.

Problem 8.1. For each symmetry above, prove the corresponding identity in Observation 2.2 by describing an appropriate involution on staircase tableaux.

Proving the second identity in this manner is easy. Namely, define a map $\iota$ by letting $\iota(\mathcal{T})$ be the tableau obtained from $\mathcal{T}$ by switching $\beta^{\prime}$ 's and $\delta$ 's, and switching $\alpha^{\prime}$ a and $\gamma^{\prime}$ 's; clearly if $\operatorname{wt}(\mathcal{T})=\alpha^{i_{1}} \beta^{i_{2}} \gamma^{i_{3}} \delta^{i_{4}} q^{i_{5}} u^{i_{6}}$, then $\operatorname{wt}(\iota(\mathcal{T}))=\alpha^{i_{3}} \beta^{i_{4}} \gamma^{i_{1}} \delta^{i_{2}} q^{i_{6}} u^{i_{5}}$. This plus Theorem 3.5 proves the second identity. It remains to find an involution $\iota^{\prime}$ proving the first identity (the remaining involution can be constructed by composing $\iota^{\prime}$ with $\iota$ ). A natural guess is to define $\iota^{\prime}(\mathcal{T})$ by transposing $\mathcal{T}$ then switching $\alpha$ 's and $\delta$ 's, and $\beta$ 's and $\gamma$ 's. This works when $q=u$, but not for $q \neq u$.

\subsection{Lifting the ASEP to a Markov chain on staircase tableaux.}

Problem 8.2. Define a Markov chain on the set of all staircase tableaux of size $n$ which projects to the ASEP in the sense of [15], such that the steady state probability of a tableau $\mathcal{T}$ is proportional to $\mathrm{wt}(T)$. Such an approach would give a completely combinatorial proof of Theorem [3.5. (This was done in [15] for $\gamma=\delta=0$.)

8.3. "Birth certificates" for particles. In the ASEP, a black particle enters from either the left (at rate $\alpha$ ) or from the right (at rate $\delta$ ). Similarly, a "hole" (or a white particle) enters from either the left (at rate $\gamma$ ) or from the right (at rate $\beta$ ). One could imagine defining a more refined ASEP, in which each particle in the lattice has attached to it its "birth certificate," that is, the information of whether it entered the lattice from the left or from the right. Such an ASEP would be a Markov chain on $4^{n}$ states (all words of length $n$ in $\alpha, \beta, \gamma$ and $\delta$ ), which projects to the ASEP upon mapping the letters $\alpha$ and $\delta$ to a black particle, and the letters $\beta$ and $\gamma$ to a white particle. One could then hope to prove an analogue of Theorem 3.5 as follows:

Problem 8.3. Fix a lattice of $n$ sites, and let $S$ be the set of all $4^{n}$ words of length $n$ on the alphabet $\{\alpha, \beta, \gamma, \delta\}$, which we think of as configurations of four kinds of 
particles - two kinds of black particles, labeled $\alpha$ and $\delta$, and two kinds of white particles, labeled $\gamma$ and $\beta$. Define a Markov chain on $S$ with the following properties:

- particles labeled $\alpha$ and $\gamma$ always enter the lattice from the left, and particles labeled $\beta$ and $\delta$ always enter from the right;

- the Markov chain projects to the ASEP;

- the steady state probability of state $\left(\tau_{1}, \ldots, \tau_{n}\right)$ is proportional to the generating function for all staircase tableaux whose border is $\left(\tau_{1}, \ldots, \tau_{n}\right)$.

8.4. A combinatorial proof of the relations of the Ansatz. In Section 6, we gave a combinatorial proof that $D, E, V, W$ satisfy relation (III) of Theorem 5.2 , by translating it into a statement about tableaux. However, we have not yet found a combinatorial proof that $D, E, V, W$ satisfy (I) and (II).

Problem 8.4. Give a combinatorial proof of relations (I) and (II) of Theorem 5.2.

We note that when $q=u$, or one of $\alpha, \beta, \gamma, \delta$ is 0 , the above problem is easy.

\subsection{Specializing our moment formula for Askey-Wilson polynomials.}

Problem 8.5. Show directly that our moment formula recovers already-known moment formulas for specializations or limiting cases of Askey-Wilson polynomials.

\section{Appendix: Staircase, Permutation, and alternative tableaux}

Definition 9.1. [44, 36] A permutation tableau $\mathcal{T}$ is a Young diagram (where rows may have length 0) whose boxes are filled with 0 's and 1's, such that each column contains at least one 1 , and there is no 0 which has simultaneaously a 1 above it in the same column and a 1 to its left in the same row. The length of $\mathcal{T}$ is the sum of its number of rows and columns.

Definition 9.2. [50] An alternative tableau $\mathcal{T}$ is a Young diagram (where rows and columns may have length 0) whose boxes are either empty or filled with left arrows $\leftarrow$ or up arrows $\uparrow$, such that all boxes to the left of $a \leftarrow$ are empty and all boxes above an $\uparrow$ are empty 3 The length of $\mathcal{T}$ is the sum of its number of rows and columns.

See [35] for more information about alternative tableaux.

Proposition 9.3. There is a bijection between staircase tableaux of size $n$ which do not contain any $\gamma$ or $\delta$, and:

(1) permutation tableaux of length $n+1$;

(2) alternative tableaux of length $n$.

\footnotetext{
${ }^{3}$ Actually the alternative tableaux of [50] were defined as Young diagrams with blue, red and empty boxes; we define them here using left and up arrows, instead of blue and red boxes, following [35.
} 
Proof. We first give a bijection from permutation tableaux to staircase tableaux. Define a restricted 0 of a permutation tableau to be a 0 which has a 1 above it in the same column. A restricted 0 is rightmost if it is the rightmost restricted 0 in its row. If $\mathcal{T}$ is a permutation tableau, we replace with a $\leftarrow$ every rightmost restricted 0 , and replace with a $\uparrow$ every 1 which is the highest 1 in its column but is not in the top row. We replace every other entry of $\mathcal{T}$ by an empty box, and delete the top row (but we remember the length of the top row by possibly inserting empty columns to the right). The result is an alternative tableau, see Figure 5 , and the map can be easily inverted.

For the second bijection, fix a staircase tableau of size $n$. For $i$ from 1 to $n$, if the $i$ th diagonal box contains an $\alpha$, then delete this entry and the column above it (this $\alpha$ will correspond to a vertical step in the south-east border of the resulting alternative tableau). Otherwise if the $i$ th diagonal box contains a $\beta$, delete this entry and the row to its left (this $\beta$ will correspond to a horizontal step in the south-east border of the resulting tableau). Then replace each $\alpha$ with an $\uparrow$ and each $\beta$ with a $\leftarrow$, and discard all the other entries. See Figure 5 .
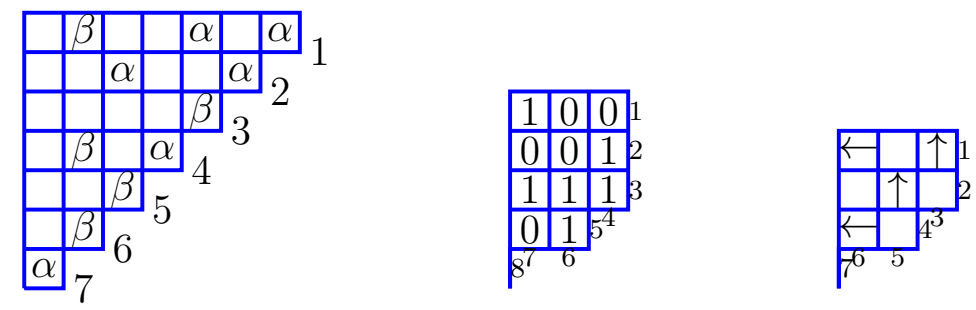

FiguRE 5. From a staircase tableau, to a permutation tableau and an alternative tableau

\section{REFERENCES}

[1] O. Angel, The stationary measure of a 2-type totally asymmetric exclusion process, J. Combin. Theory Ser. A, 113 (2006), 625-635.

[2] R. Askey and J. Wilson, Some basic hypergeometric orthogonal polynomials that generalize Jacobi polynomials, Mem. Amer. Math. Soc. 54 (1985), no. 319.

[3] J. Baik, P.L. Ferrari, S. Peche, Limit process of stationary TASEP near the characteristic line, Comm. Pure Appl. Math 63 (2010), 1017-1070.

[4] M. Balázs, T. Seppalainen, Order of current variance and diffusivity in the asymmetric simple exclusion process, Ann. Math. 171 (2010), no. 2, 1237-1265.

[5] G. Ben Arous, I. Corwin, Current fluctuations for TASEP: A proof of the Prähofer-Spohn conjecture, Ann. Prob. 39 (2011), 104-138.

[6] A. Borodin, P. Ferrari, Anisotropic KPZ growth in 2+1 dimensions: fluctuations and covariance structure, J. Stat. Mech. Theory Exp. 2009, no. 2, P02009.

[7] R. Brak, J. Essam, Asymmetric exclusion model and weighted lattice paths, J. Phys. A, 37 (2004), 1483-4217.

[8] R. Brak, S. Corteel, J. Essam, R. Parviainen, A. Rechnitzer, A combinatorial derivation of the PASEP stationary state, Elec. J. Combin. 13 (2006), R108. 
[9] R. Bundschuh, Asymmetric exclusion process and extremal statistics of random sequences, Phys. Rev. E volume 65 031911, 2002.

[10] A. Burstein, On some properties of permutation tableaux, Ann. Combin. 11 (2007).

[11] S. Corteel, Crossings and alignments of permutations, Adv. Appl. Math. 38 (2007), no 2, 149-163.

[12] S. Corteel, P. Nadeau, Bijections for permutation tableaux, European J. Combin. 30 (2009), no. $1,295-310$.

[13] S. Corteel, R. Stanley, D. Stanton, L. Williams, Formulae for Askey-Wilson moments and enumeration of staircase tableaux, Trans. Amer. Math. Soc. 364 (2012), 6009-6037.

[14] S. Corteel, L. Williams, Tableaux combinatorics for the asymmetric exclusion process, Adv. Appl. Math. 39 (2007), 293-310.

[15] S. Corteel, L. Williams, A Markov chain on permutations which projects to the asymmetric exclusion process, Int. Math. Res. Not. (2007), article ID mm055.

[16] J. de Gier and F. H. Essler, Slowest relaxation mode of the partially asymmetric exclusion process with open boundaries, J. Phys. A: Math. Theor. 41 (2008), 485002 (25pp).

[17] B. Derrida, E. Domany, D. Mukamel, An exact solution of a one dimensional asymmetric exclusion model with open boundaries, J. Stat. Phys., 69 (1992), 667-687.

[18] B. Derrida, M. Evans, V. Hakim, V. Pasquier, Exact solution of a 1D asymmetric exclusion model using a matrix formulation, J. Phys. A (1993), 1493-1517.

[19] B. Derrida, J. Lebowitz, E. Speer, Shock profiles for the partially asymmetric simple exclusion process, J. Stat. Phys. 89 (1997), 135-167.

[20] E. Duchi, G. Schaeffer, A combinatorial approach to jumping particles, J. Combin. Theory Ser. A 110 (2005), 1-29.

[21] F. Essler, V. Rittenberg, Representations of the quadratic algebra and partially asymmetric diffusion with open boundaries, J. Phys A 29 (1996).

[22] P. Ferrari, H. Spohn, Scaling limit for the space-time covariance of the stationary totally asymmetric simple exclusion process. Commun. Math. Phys. 265:1-44, 2006.

[23] P. Flajolet, Combinatorial aspects of continued fractions, Discrete Math. 32 (1980), no. 2, $125-161$.

[24] D. Foata, A combinatorial proof of the Mehler formula, J. Combinatorial Theory Ser. A 24 (1978), no. 3, 367-376.

[25] G. Gasper and M. Rahman, Basic Hypergeometric Series, second edition, Cambridge University Press, Cambridge, 2004.

[26] L. Gwa and H. Spohn, Bethe solution for the dynamical scaling exponent of the noisy Burgers equation, Phys. Rev. A 46, 844-854 (1992).

[27] M. Ismail and D. Stanton, More orthogonal polynomials as moments, Mathematical Essays in Honor of Gian-Carlo-Rota, Birkhäuser 1998, 377-396.

[28] M. Ismail, D. Stanton and X. Viennot, The combinatorics of the q-Hermite polynomials and the Askey-Wilson integral, Eur. J. Comb. 8 (1987), 379-392.

[29] K. Johansson, Shape fluctuations and random matrices, Comm. Math. Phys. 209 (2000), no. $2,437-476$.

[30] A. Kasraoui, D. Stanton and J. Zeng, The combinatorics of Al-Salam-Chihara $q$-Laguerre polynomials, to appear in Adv. Appl. Math. (2010), doi:10.1016/j.aam.2010.04.008.

[31] D. Kim, D. Stanton and J. Zeng, The combinatorics of the Al-Salam-Chihara q-Charlier Polynomials, Sem. Loth. Comb. 42 B54i (2006).

[32] R. Koekoek, P. Lesky, and R. Swarttouw, Hypergeometric orthogonal polynomials and their $q$-analogues, with a foreword by T. Koornwinder, Springer Monographs in Mathematics, Springer-Verlag, Berlin, 2010. 
[33] J. MacDonald, J. Gibbs, A. Pipkin, Kinetics of biopolymerization on nucleic acid templates, Biopolymers, 6 issue 1 (1968).

[34] A. de Medicis, D. Stanton and D. White, The combinatorics of $q$-Charlier polynomials, J. Comb. Th. A, 69 (1995), 87-114.

[35] P. Nadeau, The structure of alternative tableaux, J. Combin. Theory Ser. A 118 (2011), 16381660.

[36] A. Postnikov, Total positivity, Grassmannians, and networks, arXiv:math/0609764v1.

[37] J. Quastel, B. Valko. $t^{1 / 3}$ superdiffusivity of finite-range asymmetric exclusion processes on $\mathbb{Z}$, Commun. Math. Phys., 273:379-394, 2007.

[38] T. Sasamoto, One-dimensional partially asymmetric simple exclusion process with open boundaries: orthogonal polynomials approach, J. Phys. A 32 (1999), $7109-7131$.

[39] M. Schreckenberg, D. Wolf, Traffic and Granular Flow '97 (Singapore: Springer), 1998.

[40] L. Shapiro, D. Zeilberger, A Markov chain occurring in enzyme kinetics, J. Math. Biology 15 (1982), $351-357$.

[41] R. Simion and D. Stanton, Octabasic Laguerre polynomials and permutation statistics, J. Comp. Appl. Math. 68 (1996), p. 297-329.

[42] D. Simon, Construction of a Coordinate Bethe Ansatz for the asymmetric simple exclusion process with open boundaries, J. Stat. Mech. (2009) P07017.

[43] F. Spitzer, Interaction of Markov processes, Adv. Math. 5 1970, 246-290.

[44] E. Steingrímsson, L. Williams, Permutation tableaux and permutation patterns, Journ. Comb. Th. A, 114 (2007), 211-234.

[45] C.A. Tracy, H. Widom, Integral formulas for the asymmetric simple exclusion process, Commun. Math. Phys. 279 (2008), 815-844.

[46] C.A. Tracy, H. Widom, Asymptotics in ASEP with step initial condition, Commu. Math. Phys. 290 (2009), 129-154.

[47] C.A. Tracy, H. Widom, Total current flucturations in the asymmetric simple exclusion model, J. Math. Phys. 50 (2009), 09524.

[48] M. Uchiyama, T. Sasamoto, M. Wadati, Asymmetric simple exclusion process with open boundaries and Askey-Wilson polynomials, J. Phys. A. 37 (2004), no. 18, 4985-5002.

[49] X. Viennot, A combinatorial theory for general orthogonal polynomials with extensions and applications. Orthogonal polynomials and applications (Bar-le-Duc, 1984), 139-157, Lecture Notes in Math., 1171, Springer, Berlin, 1985.

[50] X. Viennot, slides and video from the talk "Alternative tableaux, permutations, and partially asymmetric exclusion process," at the Isaac Newton Institute, April 23, 2008, http://www. newton.ac.uk/webseminars/pg+ws/2008/csm/csmw04/0423/viennot/.

Laboratoire d'Informatique Algorithmique: Fondements et Applications, Centre National de la Recherche Scientifique et Université Paris Diderot, Paris 7, Case 7014, 75205 Paris Cedex 13 France

E-mail address: corteel@liafa.jussieu.fr

Department of Mathematics, University of California, Berkeley, Evans Hall Room 913, Berkeley, CA 94720

E-mail address: williams@math.berkeley.edu 